\title{
SETI e la grande storia: sfide ed espansione
}

Lowell S. Gustafson

Department of Political Science, Villanova University

Tradotto da Demetrio S. Yocum

Correspondence | Lowell S. Gustafson, lowell.gustafson@villanova.edu

Citation | Gustafson, L. (2020) SETI e la grande storia: sfide ed espansione. Tradotto da Demetrio S. Yocum. Journal of Big History, IV(2); 18 - 31.

DOI | https://doi.org/10.22339/jbh.v4i2.4211

\begin{abstract}
* $^{*}$
SETI è una delle sfide significative per la sua area che espande i limiti della grande storia. La grande storia aveva esercitato sulla World History un impatto simile a quello che le precedenti storie nazionali avevano avuto sulla storia religiosa. La grande storia cerca di usare le prove e le migliori analisi teoriche per integrare gli studi del cosmo, della Terra, della vita sulla Terra e dell'umanità. SETI condivide l'interesse per il cosmo, ma prende in considerazione esopianeti, astrobiologia e la possibilità di intelligenza extraterrestre. In questo articolo, si considera la relazione in via di sviluppo tra gli obiettivi di SETI e la grande storia.
\end{abstract}

\section{Introduzione}

Storie religiose

Storie politiche, nazionali e sociali

Storia mondiale

\section{Introduzione}

Il passato non è esattamente com'era una volta. Continua a cambiare. Il punto da cui noi iniziamo i nostri racconti del passato è cambiato, come pure le storie di chi prendiamo in considerazione. Questi cambiamenti avvengono attraverso un processo di sfida e di espansione e ci dicono molto su come comprendiamo noi stessi. Le storie politiche, nazionali e sociali hanno sfidato e cambiato le storie religiose e tradizionali. La World History ha sfidato e ampliato le storie nazionali. La grande storia ha fatto lo stesso con la World History. Inoltre, la ricerca di intelligenza extraterrestre (SETI) è tra le sfide che implicitamente richiedono un ampliamento della grande storia.

Uno degli aspetti che caratterizzano l'umanità è la memoria, che è diversa dalle prove del passato. Le condriti e le meteoriti che orbitano attorno al nostro sole dalla formazione del nostro sistema solare, ci forniscono prove di

\section{La grande storia \\ SETI}

Rilevazione e comunicazione

Una storia di crescita

ciò che è accaduto nello spazio oltre 4,5 miliardi di anni fa. Le rocce ignee intrusive ci danno un indizio di ciò che è accaduto milioni di anni fa sulla Terra. Ma non pensiamo che queste stesse prove possano ricordare o riflettere su ciò a cui hanno preso parte molto tempo fa.

Alcuni hanno sostenuto che le piante ricordano. ${ }^{1}$ Molti animali scimpanzé, delfini, elefanti, uccelli, ratti e altri - mostrano di ricordare il proprio passato e anche di come ciò li aiuti a prepararsi per il futuro. ${ }^{2}$ Noi individui umani non solo condividiamo la capacità di ricordare, ma possiamo anche condividere e trasmettere i nostri ricordi in un processo che David Christian ha definito apprendimento collettivo. Possiamo conservare idee riguardanti il passato anche quando ciò che ricordiamo non esiste più. Questa capacità è stata potenziata con sviluppi cognitivi che hanno dato origine al pensiero simbolico. Le scimmie Vervet

\section{Cultura popolare \\ Una storia di finali \\ Conclusioni}

emettono suoni distinti che avvertono gli altri appartenenti al loro clan della presenza e del pericolo derivato dalla presenza di aquile e leopardi. ${ }^{3}$ Quello però che non fanno è usare questi suoni in assenza del referente. Infatti di notte non si siedono attorno a un falò, con uno di loro che si scusa dicendo: "Pensavo d'aver visto un'aquila oggi quando ho dato l'allarme; e poi si è scoperto che era solo un corvo. Scusatemi per il falso allarme." L'evoluzione della capacità di mantenere un pensiero e quindi di richiamarlo esplicitamente mediante suoni precisi e comunicabili è un momento fondamentale per gli ominidi.

La memoria di tutta una serie di cose che non esiste più potrebbe sembrare spesso più importante di ciò che esiste ora. Potremmo ricordarci di coloro che ci hanno aiutato a crescere anche molto tempo dopo la loro morte. Il ricordo che noi abbiamo di loro potrebbe essere reale anche se loro non esistono

\footnotetext{
* I am grateful for the generous support from the Subvention of Publication Program of Villanova University for preparation of this article. I am also grateful to Lacy Loar-Gruenler for her editing of this article; of course any mistakes or errors are my reponsibility.
} 
più. Non c'era voluto molto per pensare che sopravvivessero sia loro che $\mathrm{i}$ ricordi che noi abbiamo di loro. I nostri ricordi sono diventati i loro spiriti. In questo, la memoria era legata a un'altra esplosione cognitiva: l'immaginazione. Siamo soliti immaginare ciò che non è mai esistito per poi far sì che tali idee diventino concrete. Possiamo supporre che nessuno abbia mai visto i propri antenati nell'aldilà, ma ci siamo dati da fare per prepararli a questa possibilità ancora prima della storia scritta. Non sappiamo se esiste veramente il paradiso, ma ci sono tombe in cui le persone sembrano aver preparato $\mathrm{i}$ loro antenati per il mondo a venire. Non solo queste persone, ma i beni di cui avrebbero avuto bisogno per vivere bene nella loro nuova vita erano dotati di spirito. E così hanno seppellito pentole, armi, gioielli e altri oggetti con loro pensando che potessero portarseli.

Un altro modo di immaginare è venuto dalla capacità di associare ricordi di ciò che avevamo visto per poter aprire la nostra mente a qualcosa di nuovo. Ne è un esempio la figurina di Löwenmensch o "uomo-leone," una scultura scoperta in Germania nel 1939 (figura 1). Ci possiamo ricordare di aver visto un leone e un uomo, ma mai una creatura che fosse entrambe le cose. La nostra capacità di immaginare una sintesi ha creato una nuova idea, una nuova alternativa di ciò che potrebbe essere. Nessuno aveva mai visto un uomo-leone, ma un artista di 35.000 ai 40.000 anni fa ha potuto immaginarlo e scolpirne uno usando l'avorio di un mammut. ${ }^{4}$ A quel tempo, nessuno aveva mai visto una città o un computer, ma alla fine sono state progettate e realizzate da persone creative.

La memoria e l'immaginazione hanno rivelato ciò che potrebbe essere reale per noi. Ci ha dato delle alternative a ciò di cui siamo stati parte nel corso del tempo, nonché su come potremmo partecipare a ciò che è accaduto prima, $\mathrm{o}$ a ciò che potrebbe accadere dopo di noi. Ciò che ricordiamo e il modo in cui riflettiamo sulla memoria ci aprono nuovi futuri.

\section{Storie religiose}

Abbiamo molti modi di procedere per tenere traccia dei nostri ricordi. Metodi per tenere traccia dei giorni e altre unità come mesi o anni potrebbero essere stati sviluppati già nel periodo neolitico. La disposizione delle pietre di Wurdi Youang risalente a 11.000 e 20.000 anni fa a Victoria, in Australia, sia servita proprio a questo scopo. Il famoso sito inglese Stonehenge, costruito circa 4.000 o 5.00o anni fa, potrebbe aver registrato e preannunciato i solstizi invernali ed estivi. Un certo numero di costruzioni Maya, come il Caracol a Chichén Itzá, sono allineate con una varietà di eventi celesti che segnano date calendariali. Questi e molti altri esempi indicano periodi prima della scrittura in cui i nostri antenati si vedevano in relazione con il cosmo, con i suoi eventi che erano accaduti e che si poteva aspettare che accadessero di nuovo. Memoria e attesa erano collegati.

È possibile che questi eventi cosmici

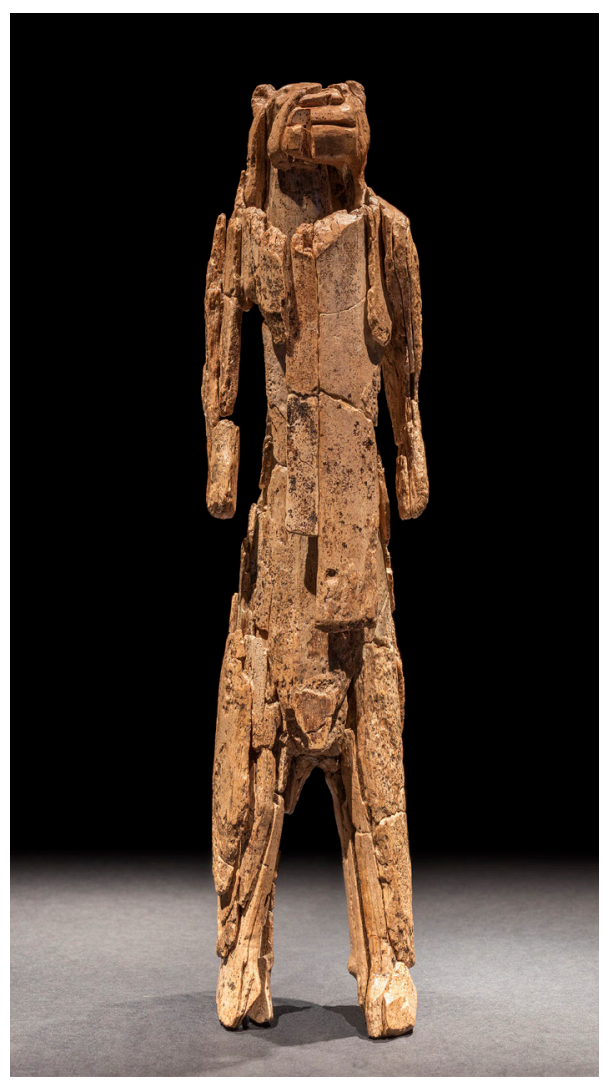

Figura 1: L'uomo-leone venissero pensati come collegati ad eventi sulla Terra, come l'apparizione di alcune piante, i movimenti di branchi d'animali o l'arrivo delle piogge. Le osservazioni cosmologiche e la loro associazione con eventi locali divennero o entrarono a far parte di rituali e di religioni. Sono stati anche utili nel tenere traccia del passare del tempo. Le religioni spesso datano il tempo a partire da un evento importante nelle loro storie o nella loro comprensione della creazione dell'universo. Ciò potrebbe principalmente avere a che fare con cicli annuali che si ripetono. Il tempo ciclico è stato spesso la norma. A volte, i cicli annuali facevano parte di cicli più lunghi. Ad esempio, i Maya calcolavano 365 giorni in un anno, (diciotto mesi ciascuno di venti giorni, con un mese di transizione di cinque giorni) o Haab': un k'atun di vent'anni, o 7.200 giorni, un b'aktun di 144.000 giorni, tredici b'aktun del lungo computo e un ciclo di 52 anni, il calendario circolare, basato su un raffronto tra il calendario rituale di 260 giorni e quello Haab'. Avevano anche altri sistemi per contare i giorni. L'antico calendario di lungo computo dei Maya iniziava dalla data più recente della creazione, l'equivalente dell'11 agosto 3114 a.C. nel calendario prolettico gregoriano.

Anche altri calendari religiosi tengono traccia del passare del tempo.

I calendari usati ufficialmente in Cina prima del 1911 erano principalmente calendari ciclici a pannelli solari che corrispondevano a eventi astronomici e stagionali utili agli agricoltori. Ci furono anche anni di regno, Huang $\mathrm{Li}$, associato a un certo numero di imperatori. Nel calendario indù sono passati 5.122 anni da quando Sri Krishna è tornato alla sua dimora eterna. Nei calendari buddisti, il Buddha ha raggiunto il parinibbāna il 13 maggio 544 a.C. circa. Yose ben Halafta, un rabbino ebreo del II secolo d.C. calcolò che la creazione era avvenuta il 25 di Elul dell'anno 1 (3761 a.C.). Maimonide, il filosofo e scienziato ebreo del XII secolo, affermò che l'inizio della creazione avvvenne nell'anno ebraico 
o (indicato come "Anno Mundi o" in latino) e che la creazione degli esserei umani avvenne in Anno Mundi 1, che corrisponde al 3761 a.C. ${ }^{5}$ L'anno in corso (2020 d.C.) nel calendario ebraico corrisponde al 5780 .

Un esempio di data tradizionale è come quella usata per pubblicazione del numero di questa rivista: 2020. Questa data si riferisce al numero di anni dalla nascita di Cristo. Sant'Agostino (354430 d.C.), nella sua opera La Genesi alla lettera (De Genesi ad litteram libri duodecim) sosteneva che la Terra avesse circa 6.000 anni. ${ }^{6}$ James Ussher, un capo della chiesa nell'Irlanda del diciassettesimo secolo, concordò all'incirca con la data di creazione ancora utilizzata per il calendario ebraico e da Agostino. La lettura da parte di Ussher di testi biblici e altri testi antichi lo portò a stabilire la data della creazione "al tramonto precedente il 23 ottobre ... del 4004 a.C." 7 Ciò che contava per i cristiani non era tanto la creazione del mondo, ma la ricreazione in Cristo. Come scritto nell'epistola della Bibbia cristiana, 2 Corinzi 5:17, ". . . se uno è in Cristo, la nuova creazione è venuta; le cose vecchie sono passate, ecco ne sono nate di nuove."

A partire dal secondo secolo dopo Cristo, alcuni vescovi nell'impero romano d'Oriente iniziarono a contare gli anni dalla nascita di Gesù. Tuttavia, vari studiosi usarono calendari diversi per stabilire che la nascita di Gesù fosse avvenuta in anni leggermente diversi. Il problema non si era ancora risolto neanche tre secoli più tardi. Dionysius Exiguus, noto anche come Dionigi il Piccolo, era un monaco originario di una zona tra l'attuale Romania e Bulgaria che in seguito fu attivo a Roma. La sua preoccupazione principale fu di stabilire le date della Pasqua in diversi anni nell'ambito del calendario liturgico annuale. Dionigi fu il primo a calcolare che c'era una data per una Pasqua 525 anni dopo la nascita di Cristo, o nell'Anno Domini, o nell'a.C., o nell'anno di nostro Signore. Alcuni avevano precedentemente affermato che Cristo era nato 5.500 anni dopo la creazione del mondo, e quindi avevano predetto che Gesù sarebbe tornato nell'anno 6ooo. "L'Era dell'Incarnazione. . . fu un metodo di computo ben presto molto usato in Italia e, in una certa misura, poco dopo anche in Spagna; durante l'ottavo e il nono secolo fu adottato in Inghilterra. Si dice che Carlo Magno sia stato il primo sovrano cristiano ad impiegarlo ufficialmente. Fu solo nel X secolo che fu utilizzato nella cancelleria papale." 9

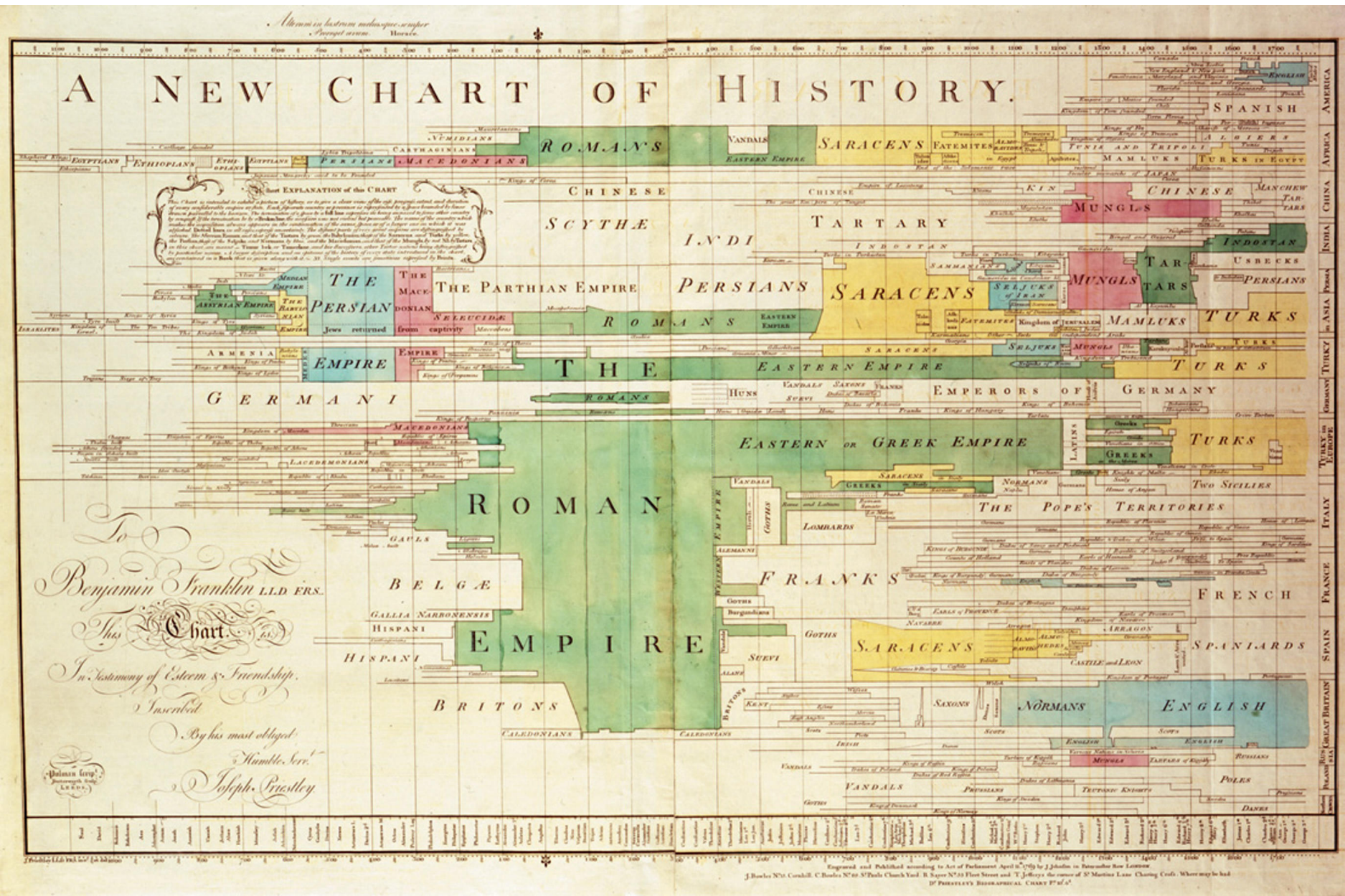

Figura 2. Una nuova carta storica. Inglese: una versione a colori di A New Chart of History di Joseph Priestly. "A Benjamin Franklin LLD. FRS. Questa carta a testimonianza di stima e amicizia. Firmato dal suo più grato e umile servitore. Joseph Priestley. 1769." 
Questo sistema di datazione ora si riferisce generalmente all'inizio di un'era comune volgare (e.v. o e.c.), o prima dell'era comune (a.C.) in luogo di quello che contava gli anni prima di Cristo, o nell'anno di nostro Signore. $\mathrm{Si}$ conserva così lo stesso numero di anni per comodità, ma vengono evitati i riferimenti a un inizio di carettere religioso.

Anche l'Islam inizia il suo calcolo degli anni da un evento fondamentale della sua storia. Il calendario islamico infatti calcola 1441 anni dall'egira (o esodo) di Maometto dalla Mecca a Medina, nota come Hijra. Da ormai molti secoli, un gran numero di persone si riconosce nel tempo all'interno di queste strutture religiose risalenti a diversi millenni prima.

\section{Storie politiche, nazionali e sociali}

Non è sempre facile distinguere tra politica e religione, poiché spesso si intrecciano. I leader politici hanno spesso rivendicato una sorta di status religioso. Tuttavia, possiamo notare come il calcolo degli anni nei vari calendari sia stato spesso connotato politicamente. Potrebbe iniziare con l'avvento al potere di un leader o di una dinastia, o in relazione a storie politiche nazionali.

Nel 1912, Sun Yat-sen annunciò che il primo gennaio di quell'anno corrispondeva al 14 Shíyīyuè 4609 dell'anno Huángdì, rendendo l'anno 1 equivalente al 2698 a.C.

Durante la Repubblica Romana, anche gli anni furono datati dai consoli. Dionigi il Piccolo voleva cambiare il sistema tradizionale, proprio del suo tempo, di contare gli anni dalla fondazione di Roma o dall'arrivo al potere di Diocleziano, cioè l'Era dei martiri.

\section{Storie nazionali}

Nella storia europea, la sovranità di Dio lasciò il posto alla sovranità dinastica dopo la Pace di Vestfalia nel 1648. Sotto il principio del Cuius regio, eius religio ("Di chi è la regione, di lui sia la religione"), i leali sudditi accettavano il cattolicesimo o il ramo del protestantesimo a cui il loro sovrano apparteneva. Essere un fedele suddito del re Enrico VIII significava essere un membro della Chiesa d'Inghilterra di cui Enrico era il capo. È soprattutto con l'Illuminismo e la Rivoluzione francese, che la sovranità dinastica lasciò il posto alla sovranità nazionale o popolare. I cittadini della nazione erano a quel tempo le persone che detenevano la sovranità politica. Le storie delle nazioni, più che le dinastie o le religioni, divennero protagoniste. Come e quando ha avuto origine e si è poi sviluppata una nazione?

Un esempio di quanto detto è Joseph

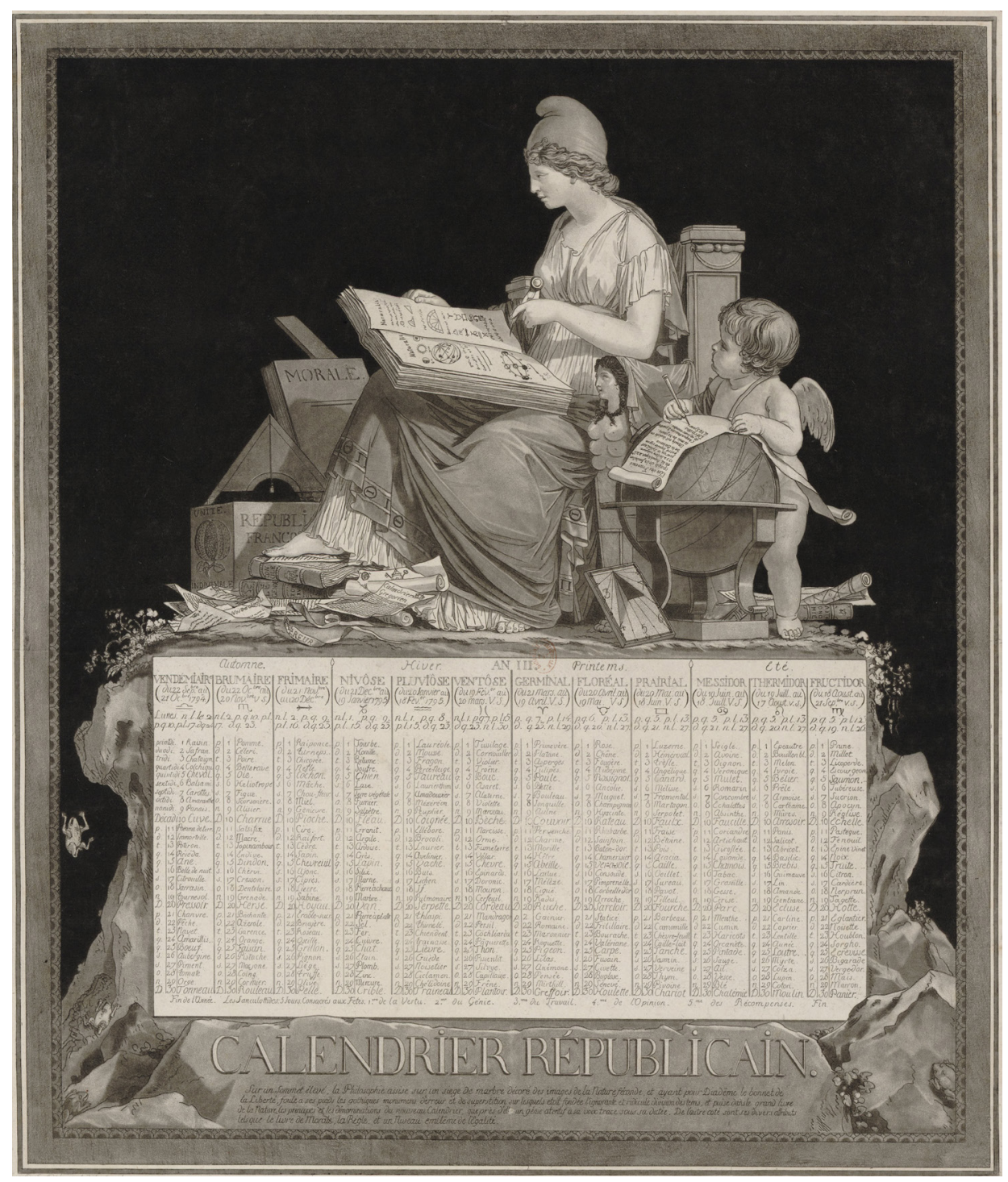

Figure 3. Calendrier républicain...: an III: [estampe] / P.L. Debucourt del. et sculp. 1794,
Priestley, teologo separatista inglese del diciottesimo secolo, filosofo naturale, chimico e teorico politico liberale. Tra i suoi risultati scientifici vi è quello di essere considerato lo scopritore dell'ossigeno. Nel 1769, dedicò la sua "la grande Carta storica" (figura 2) al suo amico e collega scienziato, anche lui politicamente attivo, Benjamin Franklin. Priestley non considerò come inizio la creazione del mondo, o un evento religioso o l'avvento al potere di un monarca, ma fece iniziare la sua storia con le antiche nazioni d'Israele ed Egitto. A dire il vero, questi erano inizi di tipo nazionali che coincidevano con il senso del tempo ereditato dalla 
Bibbia, ma il suo documento non menzionava la Genesi, Re David o il faraone Djoser. La sua nuova versione della storia era al contempo globale e nazionale; includeva molte se non la maggior parte delle grandi nazioni del mondo e alcune regioni, come la Scandinavia, la Polonia, la Russia, la Gran Bretagna, la Spagna, la Francia, l'Italia, la Turchia europea, la Turchia asiatica, la Germania, la Persia, l'India, la Cina, l'Africa e l'America.

Il Calendario repubblicano francese (figura 3) cercò di collocare il nuovo regime in Francia dopo la Rivoluzione francese del 1789 in un periodo di tempo concepito ex novo, razionale e liberato dalle superstizioni medievali. Tale calendario non faceva iniziare la cronaca del tempo con la nascita di Gesù o con l'antica Israele. Il conteggio degli anni sarebbe iniziato con gli importanti eventi recenti in Francia. Non si era però d'accordo se il conteggio degli anni sarebbe dovuto iniziare con la grande rivoluzione del 14 luglio 1789. Alla fine però coloro che favorirono l'inizio del conteggio degli anni con quello della repubblica, cioè il 22 settembre 1792 , ebbero la meglio. Il tempo decimale era considerato più razionale, quindi ogni giorno doveva avere dieci ore, ognuna con 100 minuti e ogni minuto cento secondi. I limiti alla razionalità e il rispetto della tradizione classica lasciarono il numero di anni da usare in numeri romani, come An (Anno) I o VI o X. Il calendario repubblicano fu usato in Francia per 12 anni dalla fine del 1793 al 1805 , e per 18 giorni dal Comune di Parigi nel 1871 .

L'insegnamento della storia in America non ha mai portato a un nuovo calendario, ma per molti la storia nazionale era ciò che contava. I documenti quasi sacri della nazione del 1776 e del 1787 avevano un'importanza quasi biblica. Come scrive C. Bradley Thompson, Abraham Lincoln parafrasò Proverbi (25:11) e "affermò che il principio di libertà della Dichiarazione era la parola 'detta a suo tempo' che si è rivelata per noi come 'frutti d'oro." La Costituzioneera "il 'vassoio d'argento' su cui poggiava la Dichiarazione." ${ }^{\circ}$ Non era tanto la creazione di Dio del mondo o la nuova creazione in Cristo che sembrava

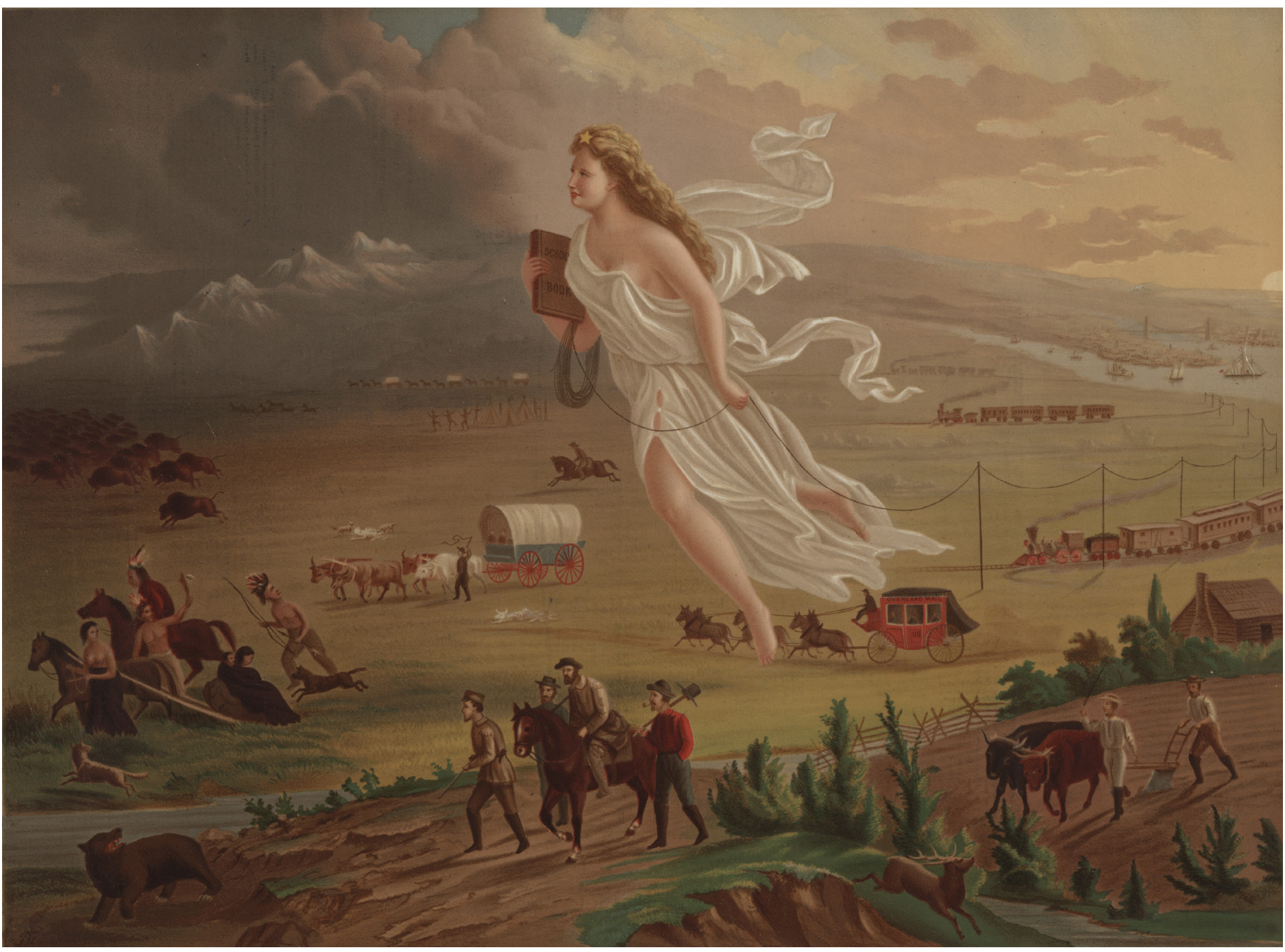

Figura 4. John Gast, American Progress, 1872. 
interessare quanto il destino manifesto della Repubblica in una terra nuova. La classica religione civile americana è il punto da dove Walter A. McDougall fece iniziare la sua Tragedy of U.S. Foreign Policy. ${ }^{11}$ Se il papato aveva la sua successione papale che risaliva a Gesù, gli Stati Uniti iniziarono la loro dinastia costituzionale con George Washington. L'attuale ultimo eletto nella linea di successione presidenziale è Donald Trump, il $45^{\circ}$ detentore di tale ufficio. Alcuni americani erano orgogliosi della successione costituzionale ininterrotta del potere che risale al 1789 .

L'identità politica americana è legata alla consapevolezza della storia dell'esperienza nazionale americana che era uno scopo fondamentale dell'educazione pubblica. Quando l'American Historical Association (AHA) fu fondata nel 1884, la storia era emersa solo di recente come disciplina accademica a parte. "I primi pochi professori nel campo della storia erano stati assunti nelle principali università negli anni settanta dell'ottocento."12 Il paese era sopravvissuto alla sua guerra civile e l'ultimo tratto della ferrovia transcontinentale era stato portato a termine nel 1869. La nazione aveva raggiunto il suo destino manifesto di integrare il territorio "da mare a splendente mare." Era quindi pronta a raccontare la sua storia. Nel $\mathbf{1 8 7 2}$, John Gast dipinse il suo "Progresso americano," che mostra una donna con fluenti capelli biondi e una tunica bianca fluttuante che vola come una dea sopra coloni e costruttori mentre occupano il territorio di animali e popoli selvaggi. (figura 4). ${ }^{3}$ Lo stato era pronto a sponsorizzare in parte le scuole pubbliche per favorire il nazionalismo e la buona cittadinanza. La storia americana era nel curriculum, ma non la storia di tutte le nazioni. Gli americani non erano i soli a celebrare la loro storia nazionale. ${ }^{14}$

Sono molte le storie di nazioni il cui scopo è incoraggiare l'identità politica nazionale. Figure eroiche, grandi battaglie ed eventi epici costituiscono le storie delle origini di molte nazioni e le identità politiche di molti cittadini. Allo stesso modo, l'American Political Science Association fu fondata pochi decenni dopo l'AHA, nel 1903. Lo studio delle scienze politiche, come la storia, era associato all'essere americano e persino alla partecipazione alla vita pubblica americana. Corsi sui tre rami del governo furono alla fine integrati da studi sulle politiche etniche, di classe e di genere, insieme a molte altre sottocategorie. Conoscere e comprendere gli eventi che hanno portato a - e i testi di - la Dichiarazione d'Indipendenza, la Costituzione, il Discorso di Gettysburg, la Lettera dal carcere di Birmingham e molti altri divenne parte dell'essere un buon cittadino americano.

Non è stata soltanto la storia politica americana ad essere un corso comunemente insegnato e una parte importante della formazione di identità nazionali. Corsi su un numero qualsiasi di altre storie nazionali, e talvolta regionali, sono stati frequenti: corsi sulla storia britannica, francese, russa, cinesi, africana, latinoamericana sono stati numerosi nelle università americane e altrove. Anche se gli studenti finivano gli studi imparando il minimo indispensabile, almeno sapevano che erano le nazioni a meritare di essere studiate. Il tempo che si copriva durante un corso variava in modo significativo. Un corso normalmente copriva diversi secoli. In alcuni casi, ad esempio i corsi sull'antica storia romana o cinese, iniziavano con eventi millenari.

\section{Storie sociali}

Nel corso della passata generazione, l'insegnamento e lo studio delle nazioni sono caduti in disgrazia. Come osserva Jill Lepore, gli intellettuali "smisero di studiare le nazioni, credendo che lo stato-nazione fosse in declino. Il mondo era cresciuto a livello globale, connesso attraverso intricate reti commerciali e da forme di trasporto e comunicazione sempre più veloci. Il futuro era cosmopolita, affermavano, non provinciale. Perché preoccuparsi di studiare le nazioni?"15

Molti storici hanno iniziato ad abbandonare le storie politiche e nazionali a favore dell'indagine sulla storia sociale o sulle storie di razza, genere, classe e altre categorie di persone nel corso di vari periodi negli ultimi decenni, secoli o millenni. Gli studi su afro-americani, afro-latini, le donne, persone appartenenti alle comunità LGBTQ e altri sono diventati molto più frequenti. A queste si sono aggiunte le storie che affrontano tematiche specifiche, come la storia ambientale. Queste sono state spesso raccontate come parte di uno sforzo per dare voceai gruppi finora marginalizzati o poco studiati. Tuttavia, la maggior parte delle storie nazionali, politiche e persino sociali erano di solito limitate al periodo della documentazione scritta del passato umano.

Ognuno ha una storia, non solo i leader politici importanti e le grandi nazioni. Se non si rientrava perfettamente in una grande narrativa, tanto meglio. Le grandi narrazioni onnicomprensive hanno finito per escludere proprio i gruppi emarginati e hanno servito gli interessi dei gruppi che dominano la creazione della cultura.

\section{Storia mondiale}

Alcuni storici hanno cercato di creare un quadro più ampio rispetto allo studio delle nazioni, dei tipi di regime o dei gruppi sociali. Poco più di un secolo dopo la formazione dell'AHA, nel 1982, alcuni storici si sono riuniti nella World History Association per raccontare una storia di globalizzazione. Queste erano intese come storie dell'umanità almeno fin dagli albori delle società agricole. ${ }^{16}$ Gli storici passano il tempo a setacciare archivi pieni di documenti primari, risalendo forse addirittura all'antica Sumer. Quando la scrittura si era sviluppata a Sumer nel 2700 a.C., c'erano comunque già diversi gruppi di persone che vivevano in tutti $i$ continenti e regioni del mondo tranne l'Antartide. I popoli parlavano lingue diverse e avevano sviluppato culture 
distinte; le ormai familiari differenze fisiche tra i popoli erano visibili. Le differenze di civiltà, regione, nazione, etnia e altre erano già ben sviluppate. L'inizio dello studio dell'uomo che si fa risalire a questo periodo finisce quindi per escludere una fase iniziale molto lunga.

Gli storici il cui scopo è promuovere un'identità politica globale affrontano il compito di cercare di costruire ponti tra varie identità culturali preesistenti. Facendo iniziare l'insegnamento della storia dagli ultimi millenni, si finisce col partire da differenze ben definite che hanno spesso portato a conflitti. Questo approccio inizia con le differenze e spesso con diffidenze e ostilità. Quale identità politica si formerebbe se gli inizi delle storie politiche venissero fatte risalire a prima delle origini delle nazioni? Se si dimostra che la storia politica umana inizia in Africa almeno 200.000 anni fa, allora nuove identità umane possono essere oggetto di corsi di studio su politiche umane oltre a quelli sulle nazioni come quella americana, britannica, cinese e altre ancora.

Può darsi che, le storie nazionali, accolte favorevolmente o meno, siano tornate di moda negli ultimi anni. Cina, India, Stati Uniti, Gran Bretagna e altri sembrano tutte desiderose di rendere di nuovo grandi le loro nazioni. Molti hanno riscontrato un nuovo tribalismo nell'ondata globale di populismo degli ultimi anni in cui vari gruppi sociali cercano confini più rigidi. Ma altri hanno sfidato e cercato di sviluppare storie nazionali, sociali e persino mondiali.

\section{La Grande storia}

William McNeill fece iniziare il suo testo di World History con la rivoluzione della produzione alimentare tra l'850o e il 7000 a.C. ${ }^{17} \mathrm{Nel}$ fare ciò, egli usò fonti diverse e non solo documentazione d'archivio. Per trattare il suo argomento, dovette fare riferimento a manufatti e all'archeologia. Ciò ha spalancato le porte ad altri storici che hanno imparato dalle scienze naturali come andare oltre il materiale scritto negli archivi per esaminare il passato che precede la scrittura. Questi storici hanno iniziato a collocare la documentazione scritta del passato umano all'interno della documentazione naturale dell'intero passato conosciuto. In tal modo, sono entrati in contatto con un mondo intellettuale che geologi, astronomi, astrofisici, chimici, biologi, antropologi fisici e altri avevano sviluppato per secoli. Furono gli scienziati naturali piuttosto che gli storici tradizionali e altri studiosi delle scienze umane e sociali a rivoluzionare la comprensione che noi abbiamo del nostro posto nel passato. Il passato non è iniziato con l'epopea di Gilgamesh in Medio Oriente circa 4000 anni fa, né con gli scritti della Bibbia o i pre-socratici. Gli scienziati hanno imparato a leggere le storie narrate da luce, pietre, ossa e sangue. E queste storie parlavano di tempi riferiti a circa milioni e miliardi di anni fa. Gli esseri umani erano effettivamente formati dagli stessi elementi sulla superficie terrestre che sono a loro comuni, ma chiaramente non erano formati direttamente da un pugno d'argilla. I primi organismi unicellulari si sono formati dagli elementi e dai composti chimici trovati sulla superficie terrestre. Gli stessi carbonio, idrogeno, aminoacidi e proteine hanno una lunga storia. Come Carl Sagan ha con evidenza osservato e in modo molto eloquente, siamo tutti fatti di materiale stellare. E poi, come hanno sostenuto Walter Alvarez e altri, il materiale stellare venne concentrato dalla Terra. ${ }^{18}$ E poi ci sono voluti miliardi di anni di evoluzione tra i primi organismi unicellulari e gli umani.

Eric Chaisson, David Christian, Fred Spier e molti altri hanno pubblicato studi documentati, dei periodi di tempo che vanno dal big bang 13,82 miliardi di anni fa, a quelli di stelle e galassie, sostanze chimiche, pianeti terrestri come la Terra, la vita, l'evoluzione e la cultura umana. La storia non è iniziata con nazioni, civiltà o persino l'agricoltura; è iniziata con l'inizio del tempo e dello spazio. ${ }^{19}$
Nel 2010, la International Big History Association si è costituita con lo scopo di esaminare "la storia integrata del cosmo, della Terra, della vita e dell'umanità, utilizzando le migliori prove empiriche disponibili e metodi accademici." Il bollettino dell'IBHA, Origins, così come il Journal of Big History della stessa organizzazione, contiene scritti e articoli accademici su questi argomenti. ${ }^{20}$ David Christian, che ha coniato il termine Big History, ha pubblicato Origin Story: A Big History of

Everything nel 2018. ${ }^{21}$ Il libro inizia con il Big Bang per arrivare all'Antropocene, cioè quel periodo attuale della storia della Terra quando gli esseri umani hanno l'impatto dominante sul pianeta.

Le storie di nazioni di tutto il mondo hanno messo in dubbio l'idea di una singola religione come la più indicata per datare tutti gli eventi. La World History ha a sua volta messo in dubbio l'idea predominante di dividere l'umanità in confini nazionali e gruppi sociali; ha perseguito invece una storia integrata di tutta l'umanità dalla rivoluzione agricola in poi. La grande storia ha a sua volta inquadrato quella storia umana nel suo passato di circa 200.000 a 300.000 anni fa, e poi i numerosi passi che sono stati fatti oltre 13.82 miliardi di anni per arrivare alla Terra, alla vita e all'umanità.

La nuova visione di chi siamo, da dove veniamo e di cosa siamo parte è stata rafforzata dalla foto di Earthrise scattata il 24 dicembre 1968 dall'astronauta William Anders dell'Apollo 8. È la foto che Fred Spier ha usato per la copertina del suo libro Big History and the Future of Humanity. Ė da allora che egli riflette su quella foto. ${ }^{22}$ Vedere la Terra dal punto di vista della luna ha cambiato chi siamo. La bellissima Terra blu, bianca e verde mostrata come un gioiello in uno spazio molto oscuro e inquietante. Non c'è pianeta B verso cui rifugiarsi con la nostra tecnologia, né allora né ora, se finissimo col rendere la nostra Terra inabitabile. Per molti, le vecchie idee di sicurezza nazionale impallidivano di fronte alla sicurezza 


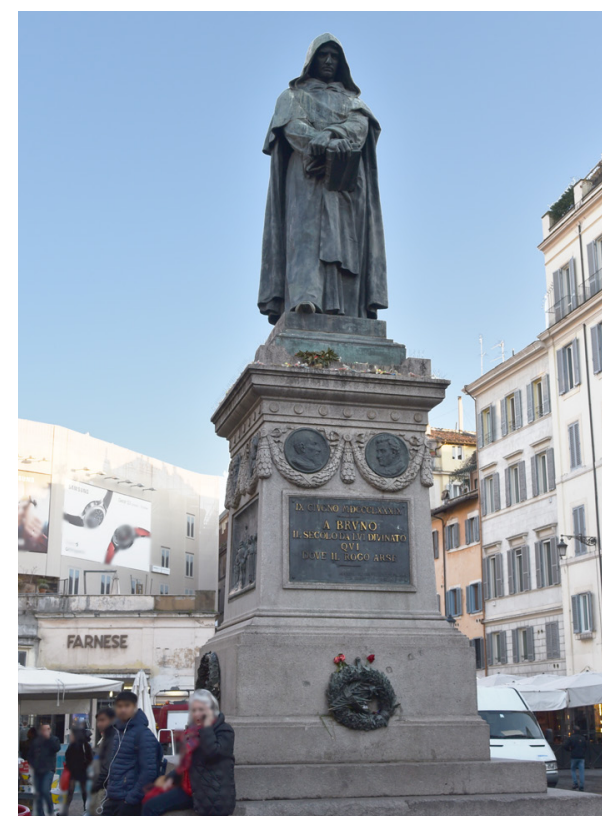

Figura 5: Luogo a Roma dove il 17 febbraio 1600 Giordano Bruno fu bruciato sul rogo per le sue posizioni eretiche. Foto di Lowell Gustafson.

della patria globale.

\section{SETI e una storia di crescita}

Per quanto sorprendente fosse la vista della Terra dalla luna, molti si chiedevano se ci fossero altri pianeti abitabili oltre il nostro sistema solare. Una volta si era pensato che Marte fosse una possibile patria per la vita intelligente, ma si è sempre finito col mettere in dubbio che potesse avere le capacità di ospitare persino la vita microbica. Una delle nostre migliori speranze è nei mari di una delle lune di Giove, Io. La ricerca di esopianeti (pianeti al di fuori del nostro sistema solare), la ricerca di vita in qualsiasi parte dell'universo oltre quella sulla Terra come pure la ricerca di intelligenza extraterrestre sfida e prolunga la grande storia. La grande storia non èabbastanza grande per questi campi. Esistono molti pianeti oltre alla Terra; a partire dal 2019 ne sono stati rilevati oltre 4.0oo proprio nell'area limitrofa della nostra galassia. ${ }^{23}$ Il numero di pianeti nell'universo con oltre 100 miliardi di galassie deve essere strabiliante.

L'interesse nella possible esistenza di molti altri pianeti non è solo tipico dei nostri tempi. Nel quindicesimo secolo, il cardinale tedesco Nicola Cusano propose l'esistenza di altri pianeti attorno ad altre stelle oltre a quelli intorno al nostro sole. Egli scrisse che "La terra è una stella come le altre stelle, non è il centro dell'universo, non è a riposo, né i suoi poli sono fissi. I corpi celesti non sono necessariamente sferici, né le loro orbite sono circolari."24 Il frate domenicano italiano del XVI secolo, Giordano Bruno, sosteneva che le stelle fossero soli lontani circondati dai loro stessi pianeti, così come l'universo è infinito e non poteva quindi avere un centro.

Nel 1685, l'autore francese Bernard le Bovier de Fontenelle pubblicò le Conversazioni sulla pluralità dei mondi. Non lo scrisse in latino, pratica comune per gli studiosi dell'epoca, ma in francese poiché il suo scopo era quello di rendere le idee accessibili alla cultura popolare. ${ }^{25}$

Una differenza notevole tra questi primi scritti e SETI è rappresentata dai grandi progressi scientifici e tecnologici compiuti negli ultimi tempi che ci consentono di cercare prove fisiche di esopianeti piuttosto che speculare soltanto su di essi. Ora possiamo usare l'osservazione per stabilire che ci sono altri pianeti abitabili, cioè quelli con acqua liquida che possono ospitare la vita così come possiamo immaginarla.

Abbiamo trovato con successo prove di esopianeti; stiamo ancora cercando prove di qualsiasi altra vita, intelligente o meno, oltre la Terra. È probabile che troveremo prove perché è ancora una domanda aperta. Nel 1961, Frank Drake scrisse la sua famosa equazione che proponeva ciò che dovevamo sapere prima di poter calcolare quante forme di vita oltre la Terra esistevano. La formula era:

$$
\begin{aligned}
& N=R_{*} \cdot f_{\mathrm{p}} \cdot n_{\mathrm{e}} \cdot f_{\mathrm{l}} \cdot f_{\mathrm{i}} \cdot f_{\mathrm{c}} \cdot L \\
& \text { dove }
\end{aligned}
$$

$\mathrm{N}=$ è il numero di civiltà nella nostra galassia con cui potrebbe essere possibile comunicare (cioè che sono sul nostro attuale cono di luce passato);

e
$R_{*}=$ al tasso medio di formazione stellare nella nostra galassia

$f_{\mathrm{p}}=$ alla frazione di quelle stelle che hanno pianeti

$n_{\mathrm{e}}=$ al numero medio di pianeti che possono potenzialmente sostenere la vita, per stella che ha pianeti

$f_{1}=$ alla frazione di pianeti che potrebbe sostenere la vita e che effettivamente sviluppa la vita ad un certo punto

$f_{\mathrm{i}}=$ alla frazione di pianeti con trace di vita che effettivamente continua a sviluppare la vita intelligente (civiltà)

$f_{\mathrm{c}}=$ alla frazione di civiltà che sviluppano una tecnologia che rilascia nello spazio segni rilevabili della loro esistenza

$L=$ al periodo di tempo durante cui tali civiltà rilasciano segnali rilevabili nello spazio

Sulla Terra soltanto, c'è quasi un'infinita varietà di forme di vita oltre agli umani. Sarebbe così sorprendente se sul numero quasi illimitato di pianeti nell'universo ci fossero molte altre forme di vita, comprese quelle intelligenti? L'interesse della grande storia per il cosmo va bene; la sua enfasi sulla Terra, la vita sulla Terra e l'umanità è però troppo limitato. Dobbiamo anche pensare, e cercare, esopianeti, astrobiologia ed esointelligenza.

A molti sembrava molto probabile che, con innumerevoli pianeti abitabili, di cui abbiamo scoperto solo alcuni, dovessero esserci molte forme di vita extraterrestre. $^{26}$ Lo pensava anche il grande fisico italo-americano, Enrico Fermi, ma poi si chiese: "Ma allora, dove sono tutti?"27

\section{Rilevazione e comunicazione}

Finora non abbiamo ancora rilevato alcun tipo di vita o intelligenza extraterrestre. Una speranza ancora più ambiziosa è poter forse comunicare un giorno con qualche forma di vita extraterrestre o formare una sorta di relazione con altri esseri. ${ }^{28} \mathrm{E}$ ancor più ambiziosa è l'idea che possano visitare la Terra o per noi di visitare loro. ${ }^{29}$ Date le enormi difficoltà di percorrere 
tali distanze, di certo non abbiamo modo di farlo adesso o nel prossimo futuro. Altre civiltà molto più avanzate potrebbero averlo capito, ma non ci sono ancora prove che abbiano convinto gli scienziati di tali visite. Non abbiamo neanche rilevato gli irraggiamenti molto più probabili di civiltà oltre la Terra.

\section{Una storia di crescita}

Anche se non abbiamo trovato esempi di vita semplice o intelligente oltre la Terra, tale ricerca è importante. Per molti secoli, abbiamo pensato che la nostra Terra fosse il centro dell'universo. Pensavamo di essere le uniche forme di vita nel cosmo. Anche solo un secolo fa, ben dopo quindi aver appreso che orbitavamo attorno al sole, la maggioranza pensava che fossimo nell'unica galassia dell'universo. Solo quando Edwin Hubble e altri hanno dimostrato che esistevanoun numero enorme di altre galassie ci siamo resi conto che non c'erano solo poche nuvole o nebulose che circondavano la Via Lattea. Prima del 1995, non avevamo prove di pianeti al di fuori del nostro sistema solare. Ora sappiamo che ce ne sono migliaia in poche migliaia di anni luce dai nostri. Non abbiamo dato per scontato che siamo tutto ciò che esiste. Abbiamo cercato e trovato altri pianeti. Abbiamo cercato la vita oltre la Terra e la vita intelligente oltre la Terra - e stiamo ancora cercando.

Immaginare e cercare la vita e l'intelligenza extraterrestre rappresenta il livello successivo di complessità che la grande storia non aveva messo in evidenza prima. Il racconto della grande storia riguardava già la crescente complessità delle relazioni. La storia dipendeva dall'emergere di relazioni precedenti incorporate in nuove relazioni più complesse con nuove caratteristiche. La grande storia ha presentato una narrazione di quark "su" e "giù" nelle relazioni tra neutroni e protoni attraverso la mediazione della forza forte. Protoni e neutroni hanno formato relazioni con elettroni attraverso la mediazione della forza elettromagnetica. L'equilibrio dinamico tra gravità e fusione nucleare ha permesso alle stelle di formare nuclei con un numero maggiore di protoni rispetto a quanto precedentemente ottenuto dall'idrogeno e dall'elio. Questi elementi, una volta espulsi nello spazio per mezzo di stelle morenti o supernove, si collegavano all'interno di sostanze chimiche multi-elemento. Con questo processo, oltre alle stelle di neutroni che si scontrano tra loro, si ebbero elementi più pesanti per formare pianeti terrestri. Alcuni come la Terra avevano più livelli, con un nucleo metallico, il magma, un fondo marino, le piastre continentali e molto altro. La chimica si è evoluta in biochimica che si è evoluta negli insiemi di relazioni più complesse fino ad oggi all'interno di virus e cellule procarioti unicellulari. Queste alla fine sono diventate ancora più complesse con nuovi organelli e un nucleo nelle cellule eucariote. Le forme di vita multicellulari sono esplose in un numero quasi infinito di forme di vita nel periodo Cambriano. Una devastante meteora potrebbe aver causato l'estinzione dei dinosauri nonaviari e aver dato spazio ai mammiferi per evolversi infine in homo sapiens. Le relazioni tra individui della nostra specie andavano dai gruppi nomadi imparentati tra loro, ai villaggi agricoli stanziali, alle città stato, alle nazioni, agli imperi e alle fragili istituzioni globali. Ora, con autori come H. G. Wells e gli scienziati SETI, potremmo immaginare un livello successivo di relazioni interplanetarie e intergalattiche. L'idea era tanto assurda quanto l'idea di una cellula procariota per i primi atomi di carbonio, idrogeno e ossigeno.

C'è un che di ironico nel modo in cui comprendiamo noi stessi, la vita e la Terra non appena iniziamo a pensare ad altri pianeti e a forme di vita ed esseri intelligenti. Anche se non troviamo la vita extraterrestre, la ricerca ci fa crescere. Pensiamo alle origini di altri pianeti, ad altre forme di vita, ad altri esseri intelligenti e così non pensiamo esclusivamentealle nostrestesseorigini.
Questo processo non è una storia delle origini per noi; è una storia di crescita. Si potrebbe dire che una persona diventa maggiorenne con la consapevolezza che esistono indipendentemente altre persone che hanno le proprie idee, le proprie emozioni e i propri interessi. Alcuni hanno raggiunto tale maturità da un vivo interesse per l'evoluzione di altre specie qui sulla Terra. La linea che inizia dalla vita non si estende solo all'umanità, come nella dichiarazione di intenti dell'IBHA, ma a un numero quasi infinito di altre specie sulla e forse oltre la Terra.

\section{Cultura popolare}

Molte persone non hanno aspettato la semplice prova della vita e dell'intelligenza extraterrestre per immaginare cosa potrebbe essere e come potrebbe influenzarci. Le forme di vita e le civiltà altamente sviluppate su altri pianeti hanno attirato la nostra attenzione attraverso una serie di fonti immaginarie, tra cui romanzi, film, fumetti, serie televisive e canzoni.

Percival Lowell rese popolare l'idea della vita intelligente su Marte nei suoi tre libri: Mars (1895), Mars and Its Canals (1906), e Mars as the Abode of Life (1908).30 Nel giro di due anni dall'uscita del libro di Lowell del 1895 , H. G. Wells pubblicò il suo romanzo La guerra dei mondi. Nel 1938, Orson Welles lo adattò e ne fece recitare la storia alla radio, creando un enorme panico in tutti gli Stati Uniti. ${ }^{31}$

Gli avvistamenti di UFO erano più comuni negli anni ' 50 , ma ne continuiamo a ricevere regolarmente segnalazioni, con il sospetto che il nostro governo stia sopprimendo la conoscenza su di essi per paura del panico che causerebbe..$^{22}$

Nel 1966, i Byrds cantarono in "Mr. Spaceman" di:

... quegli alieni che vengono ogni notte

Quelle luci a forma di disco volante mettono le persone in difficoltà

Lasciano impronte verde blu che brillano nel buio 
Spero che tornino a casa sani e salvi.

La serie televisiva Star Trek è andata in onda dal 1966 al 1969, e sin da allora ha avuto un folto numero di devoti fan. La serie di film di grande successo di George Lucas, Star Wars, è iniziata nel 1977. Nel 1982, abbiamo visto il magnifico film di Steven Spielberg, E.T. l'Extra-Terrestre. Nel 1997, Jodie Foster ha recitato in Contact, basato sul libro di Carl Saga dallo stesso titolo. Non meno incantevole è stato Avatar del 2009 di James Cameron. La lista di racconti popolari sulla vita extraterrestre è lunga.33 Pare che non possiamo fare a meno della paura, della meraviglia, dell'eccitazione e dello stupore che deriva dal pensiero di ciò che le forme di vita su altri pianeti potrebbero significare per noi.

Mentre tali documenti sono spesso incredibilmente divertenti, spesso ci permettono anche di riflettere su ciò che la scoperta della vita extraterrestre significherebbe per noi. Andremo in bicicletta con E.T. attraverso il cielo o lotteremo contro Darth Vader in battaglie inter-galattiche ad alta tecnologia? Le forme di vita extraterrestre che scopriremo saranno solo microbi? Ci offriranno nuove medicine o porteranno malattie pandemiche? Come immaginiamo il nostro rapporto con loro? Ci sono buone ragioni per essere affascinati dalla vita extraterrestre ancora da scoprire.

\section{Una storia di finali}

Crescere non dipende solo da una relazione, anche se solo speculativa, con forme di vita da altri pianeti. Proviene anche da un senso di mortalità. Anche se rispondiamo al paradosso di Fermi e troviamo prove di altre forme di vita intelligenti attraverso il rilevamento di una sorta di segnali radio, è probabile che le nostre nuove forme di vita trovate siano molto distanti da noi. Abbiamo cercato solo pianeti abitabili entro 5.000 anni luce. Solo la nostra galassia ha 100.000 anni luce di diametro. Abbiamo appena iniziato a esplorare la nostra galassia, e ancor meno gli altri cento miliardi. Se riceviamo un segnale da soli mille anni luce, ci vorranno al massimo 2000 anni prima che riusciamo a mandare loro la nostra risposta e quindi ricevere poi la loro. Questo non è certo la frequenza di comunicazione su cui costruire una relazione significativa. Chiunque avrà sentito il loro primo segnale e poi avrà inviato una risposta sarà sicuramente morto prima di ricevere una risposta. Ancora più problematico è non sapere se la loro civiltà esisterà ancora quando finalmente saremo riusciti a far arrivare la nostra risposta. Forse anche loro stanno distruggendo il proprio ambiente o si stanno facendo saltare in aria a vicenda. Esisterà la nostra di civiltà quando riceveremo la loro risposta? Dopo solo una manciata di scambi di messaggi con i nostri nuovi amici, è più probabile che dovremmo ammettere che la nostra specie si sarà estinta.

Certo, anche la grande storia non ha a che fare solo con origini; ha avuto anche a che fare con finali. Abbiamo ascoltato gli astronomi e gli astrofisici che hanno parlato di come il nostro sole diventerà un gigante rosso entro cinque miliardi di anni, espandendosi fino a quando non farà evaporare gli oceani della Terra e friggere qualsiasi creatura ancora aggrappata alla vita. Il colpo di grazia verrà dall'energia oscura che sta separando le galassie nel nostro universo. Molti continuano a svanire oltre un orizzonte degli eventi, per non essere mai più visti da noi. Con abbastanza tempo, la maggior parte delle galassie nel nostro universo si sarà allontanata dalla nostra vista, lasciandoci con un cielo prevalentemente nero. E poi, le nostre galassie vicine e persino la materia potrebbero disgregarsi. Chinua Achebe aveva più ragione di quanto potesse sapere: le cose davvero vanno in pezzi. ${ }^{34}$ $\mathrm{O}$ le cose vengono fatte a pezzi. Alla fine proprio tutto. In questa prospettiva, il Big Rip, o Grande Strappo, segue il Big Bang. Non solo noi come persone e come specie finiremo, finirà anche il nostro sistema solare e il nostro intero universo - forse. Sapevamo di questa possibilità, ma Origins continuava a sembrare più ottimista. Forse molti di noi, piuttosto avanti negli anni, si comportano come adolescenti temerari che pensano di essere immortali. Se concludiamo la nostra storia con l'Antropocene, sembra quasi che questo sia un periodo che durerà per sempre. Non lo sarà; non sarà così. L'Homo sapiens esiste da circa due o trecentomila anni. Per quanto tempo riusciremo a sopravvivere nessuno lo sa, ma è altamente improbabile che saremo qui fino a quando il nostro sole raggiungerà lo stadio di gigante rosso che renderà impossibile la vita sulla Terra. La speranza che dopo aver completato la distruzione del nostro pianeta natale possiamo migrare su un altro è un'idea contemplata da alcuni. Per ora è roba da fantascienza. Discutere su come finiranno l'umanità, la vita, la Terra e gli esopianeti non è certo divertente, ma fa parte della nostra storia di crescita.

\section{Conclusioni}

SETI pone una sfida e prolunga i limiti della grande storia esaminando il pianeta Terra, la vita sulla Terra e l'umanità. Ci ricorda che esiste una miriade di altre forme di vita sulla Terra e che ne possono esistere altre su altri pianeti. Gli Homo sapiens - "i saggi" - potrebbero essere solo uno tra molti tipi di esseri intelligenti, ognuno con le proprie storie lunghe e complesse. In un certo senso, abbiamo ripreso la nostra posizione al centro dell'universo che occupavamo nella visione del mondo tolemaico. Solo che ora anche tutti gli altri pianeti e le galassie del nostro universo hanno avuto il proprio inizio nello stesso punto, e che dalla loro prospettiva, tutto il resto si sta espandendo dalla loro posizione. Ogni punto nell'universo è il centro dell'universo. Ogni luogo - e ogni forma di vita - ha una sua storia che risale all'inizio del tempo. Il Big Bang conduce a noi e a tutto il resto. Lo studio della Terra, della vita e dell'umanità deve far parte dello studio 
degli esopianeti, dell'astrobiologia, di tutta la vita possibile e di tutti i modi di essere intelligente. Questo sforzo può aiutarci a scoprire e amare il prossimo.

\section{Endnotes}

1 Sarah Abbott, "Plant Memory," Untamed Science, https:// untamedscience.com/biology/

plants/plant-memory/, last accessed December 18, 2019; Gagliano M et al. 2014. L'esperienza insegna alle piante ad apprendere più velocemente e dimenticare più lentamente negli ambienti in cui è importante. Oecologia, pubblicata online il 5 gennaiozo14; doi: 10.1007/so0442-013-2873-7; Robert Krulwich, "Can a Plant Remember? This One Seems to - Here's the Evidence," National Geographic, December 15, 2015, https://www. nationalgeographic.com/science/ phenomena/2015/12/15/can-aplant-remember-this-one-seemsto-heres-the-evidence/; Sarah Laskow, "The Hidden Memories of Plants, Atlas Obscura, September 5, 2017, https://www.atlasobscura. com/articles/plant-memory-

hidden-vernalization; ultimo accesso 18 dicembre 2019.

2 Frans de Waal, "The Surprising Complexity of Animal Memories" The Atlantic, June 2, 2019, https://www.theatlantic.com/ science/archive/2019/06/ surprising-complexity-animalmemories/589420/; ultimo accesso 17 dicembre, 2019; Panoz-Brown et al., 2018, "Replay of Episodic Memories in the Rat," Current Biology 28, 1628-1634, https:// doi.org/10.1016/j.cub.2018.04.006; ultimo accesso 17 dicembre, 2019; Christine Dell-Amore, Dolphins have the longest memories in the animal kingdom, August 6, 2013, National Geographic, https:// www.nationalgeographic.com/ news/2013/8/130806-dolphinsmemories-animals-sciencelongest/; ultimo accesso 17 dicembre 2019.

3 Klaus Zuberbühler, PredatorSpecific Alarm Calls in Campbell's Monkeys, Cercopithecus campbelli, Behavioral Ecology and Sociobiology, Vol. 50, No. 5 (Oct., 2001), pp. 414-422, https://www. jstor.org/stable/4601985; ultimo accesso 16 dicembre 2019.

4 The Lion Man: an Ice Age masterpiece, The British Museum, https://blog.britishmuseum. org/the-lion-man-an-ice-agemasterpiece/; ultimo accesso 16 dicembre 2019.

5 David B. Green, "This Day in Jewish History 3761 BCE: The World Is Created, According to the Hebrew Calendar and an Obscure Sage. Basing himself on no source but the bible, Rabbi Yose ben Halafta, who lived in the and century CE, sat down and did the math" Haaretz, Oct 07, 2015, ultimo accesso 10 dicembre 2019. https://www. haaretz.com/jewish/3761-bce-theworld-is-created-1.5405777.

6 Taylor, J.H., traduzione e commento ad Agostino, The Literal Meaning of Genesis (Augustine, De Genesi ad Litteram), Vol. 1, Newman/Paulist Press, New York, 1982. Si veda inoltre Augustine, The City of God, 12.10, 'Of the falseness of the history which allots many thousand years to the world's past', in Schaff, P. (Ed.), NPNF1-02, ref 6, pp.232-233.

7 Ussher, James. Annales Veteris Testamenti, a prima mundi origine dedvcti: una cum rerum asiaticarum et cegyptiacarum chronico, a temporis historici principio usque ad Maccabaicorum initia producto. Londini, ex officina J. Flesher, \& prostant apud J. Crook \& J. Baker, 1650. Pdf. https://www.loc.gov/ item/21002221/. https://www.loc. gov/resource/rbctos.2017gen52659.

8 Declercq, Georges: Anno Domini. The Origins of the Christian Era. Turnhout Belgium. 2000.

9 Lersch, Chronologie, Freiburg, 1899, p. 233, citato in "Dionysius Exiguus," the Catholic Encyclopedia, http://www.newadvent.org/ cathen/o501ob.htm, ultimo accesso 13 dicembre 2019.

10 Thompson, C. Bradley. America's Revolutionary Mind (p. xi). New York, Encounter Books, 2019.

11 McDougall, Walter A.. The Tragedy of U.S. Foreign Policy: How America's Civil Religion Betrayed the National Interest. New Haven, Yale University Press, 2016, p. 25.

12 "Brief History of the A HA," American Historical Association, ultimo accesso 17 dicembre 2019, http://www.historians.org/aboutaha-and-membership/aha-historyand-archives/brief-history-of-theaha.

13 John Gast, American progress, Library of Congress Prints and Photographs Division Washington, D.C. 20540 USA http://hdl.loc. gov/loc.pnp/pp.print, ppmsca 09855 http://hdl.loc.gov/loc.pnp/ ppmsca.09855, Reproduction Number: LC-DIG-ppmsca-09855 Library of Congress Prints and Photographs Division Washington, 
D.C. 20540 USA http://hdl.loc.gov/ loc.pnp/pp.print. Ultimo accesso 17 dicembre 2019 .

14 Benedict Anderson, Imagined Communities: Reflections on the Origin and Spread of Nationalism (London: Verso,1991); Marc Ferro, The Use and Abuse of History: Or How the Past Is Taught to Children (London: Routledge, 2003); Ernest Gellner, Nations and Nationalism (Ithaca: Cornell University Press, 1983); Stephen J. Hartnett, Lisa B. Keranen, and Donovan Conley, eds., Imagining China: Rhetorics of Nationalism in an Age of Globalization (East Lansing, Michigan: Michigan State University Press, 2017); Derek Hastings, Nationalism in Modern Europe: Politics, Identity and Belonging since the French Revolution (London; New York: Bloomsbury Academic, 2017); Guntram Henrik Herb and David H. Kaplan, eds. Scaling Identities: Nationalism and Territoriality (Lanham, Maryland: Rowman \& Littlefield, 2018); John Hutchinson, Nationalism and War (New York: Oxford University Press, 2017); Fawcett Kohl, eds. Nationalism, Politics and the Practice of Archaeology (Cambridge University Press, 1996); Ronald Grigor Suny, "Constructing Primordialism: Old Histories for New Nations," The Journal of Modern History 73, no. 4 (December 2001): 862-896.

15 Jill Lpore, This America: The Case for the Nation, New York, Liveright Publishing Corporation, 2019, p.15.

16 Un classico del genere è William $\mathrm{H}$. McNeill, A World History, Oxford, Oxford University Press, 1979.

17 William H. McNeill, A World History, Oxford, Oxford University Press, 1967.

18 Olga García-Moreno, Luís Erick Aguirre-Palafox, Walter Álvarez, William Hawley. "A Little Big History of Iberian Gold," Journal of
Big History, Volume 1, numero 1, 2017, https://doi.org/10.22339/jbh. vii1.2243.

19 Si veda ad esempio: Craig Benjamin, Esther Quaedackers, David Baker, The Routledge Companion to Big History, Nw York, Routledge, 2020; Brown, Cynthia Stokes. Big History: From the Big Bang to the Present. New York: New Press: Distributed by W. W. Norton, 2007; Carroll, Sean. The Big Picture: On the Origins of Life, Meaning, and the Universe Itself. New York: Dutton, 2017; Chaisson, Eric. Epic of Evolution: Seven Ages of the Cosmos. New York: Columbia University Press, 2006; Christian, David. Maps of Time: An Introduction to Big History. The California World History Library. Berkeley: University of California Press, 2004; Christian, David, Cynthia Stokes Brown, and Craig Benjamin. Big History: Between Nothing and Everything. New York: McGraw Hill Education, 2014; Dartnell, Lewis. Origins: How Earth's History Shaped Human History, New York, Basic Books, 2019; Hazen, Robert. Genesis: The Scientific Quest for Life's Origin. Washington, DC: Joseph Henry Press, 2005; Hazen, Robert. The Story of Earth: The First 4.5 Billion Years, from Stardust to Living Planet. New York: Viking, 2012; Rodrigue, Barry, Leonid Grinin, Andrey Korotayev, co-editors, From Big Bang to Galactic Civilizations: A Big History Anthology. Delhi: Primus Books, 2015-2016. threevolumes; Shubin, Neil. The Universe Within: Discovering the Common History of Rocks, Planets, and People. New York: Pantheon Books, 2013; Shubin, Neil. Your Inner Fish: A Journey into the 3.5-Billion-Year History of the Human Body. New York, Vintage, 2008; Spier, Fred. Big History and the Future of Humanity. Chichester, West Sussex, UK: John Wiley \& Sons Inc., 2015; Volk, Tyler, Quarks to Culture: How We Came to
Be, New York, Columbia University Press, 2017.

20 Numeri passati di Origins sono disponibili all'indirizzo https:// bighistory.org/members/originsbulletin/; il Journal of Big History è disponibile qui: https://jbh. journals.villanova.edu/.

21 David Christian, Origin Story: A Big History of Everything, New York, Little, Brown and Company, 2018.

22 Spier, F. (2019) Sull'impatto sociale, o meno, della foto, si veda Journal of Big History, III (3); 117 - 150. https:// doi.org/10.22339/jbh.v3i3.3390, ultimo accesso 15 dicembre 2019.

23 Dennis Overbye, Search for Habitable Worlds Joined by New European Space Telescope, New York Times, December 18, 2019, https:// www.nytimes.com /2019/12/18/ science/cheops-satellite-launch .html?action=click\&module= RelatedLinks\&pgtype=Article, ultimo accesso 23 dicembre 2019; Exoplanet Exploration: Planets beyond our solar system, NASA, https://exoplanets.nasa.gov/, ultimo accesso 19 dicembre 2019; Campante, Tiago L., Nuno C. Santos, and Ma'rio J. P. F. G. Monteiro. Asteroseismology and Exoplanets: Listening to the Stars and Searching for New Worlds: IVth Azores International Advanced School in Space Sciences. Cham: Springer, 2018; Carroll, Michael. Earths of Distant Suns: How We Find Them, Communicate With Them, and Maybe Even Travel There. Cham: Springer International Publishing: Imprint: Copernicus, 2017; Exoplanet Discoveries: Have We Found Other Earths?: Joint Hearing Before the Subcommittee On Space \& Subcommittee On Research, Committee On Science, Space, and Technology, House of Representatives, One Hundred Thirteenth Congress, First Session, Thursday, May 9, 2013. Washington: 
U.S. Government Printing Office, 2013; Fontenelle, M. de 1657-1757., and John Glanvill. A Plurality of Worlds. London: Printed for R. Bentley and S. Magnes, 1688; Frank, Adam. Light of the Stars: Alien Worlds and the Fate of the Earth. New York, NY: W.W. Norton \& Company, 2018; Howard, Sethanne. "Exoplanets." Journal of the Washington Academy of Sciences 97, no. 3 (2011): 33-53; Kitchin, C. R. Exoplanets: Finding, Exploring, and Understanding Alien Worlds. New York, NY: Springer, 2012; Lemonick, Michael D. Mirror Earth: The Search for Our Planet's Twin. 1st U.S. ed. New York: Walker, 2012; Sage, Leslie. "Exoplanets." Nature 513, no. 7518 (2014): 327; Seager, Sara. Exoplanets. Tucson: University of Arizona Press, 2011.

24 Nicholas of Cusa, Catholic Encyclopedia, https://www. catholic.org/encyclopedia/ view.php?id=8455, last accessed December 18, 2019; Nicholas of Cusa (1401-1464); si veda anche The Internet Encyclopedia of Philosophy (IEP), https://www.iep.utm.edu/ nicholas/\#H4, ultimo accesso 18 dicembre 2019 .

25 A plurality of worlds written in French by the author of the Dialogues of the dead; tradotto in inglese da Glanvill. Fontenelle, M. de (Bernard Le Bovier), 1657-1757., Glanvill, John, 1664?-1735. London: Printed for R. Bentley and S. Magnes, 1688. https://quod.lib.umich.edu/e/ eebo/A39871.00o1.001?view=toc, ultimo accesso 18 dicembre 2019.

26 Asimov, Isaac. Extraterrestrial Civilizations. New York: Crown Publishers, 1979; Dick, Steven J. Plurality of Worlds: The Origins of the Extraterrestrial Life Debate From Democritus to Kant. Cambridge [Cambridgeshire]; New York: Cambridge University Press, 1982; Dick, Steven J. Life On Other Worlds:
The 2oth-century Extraterrestrial Life Debate. Cambridge ; New York: Cambridge University Press, 1998; Hamilton, Joseph. The Starry Hosts: A Plea for the Habitation of the Planets. London: Edinburgh: Simpkin, Marshall; A. Elliott, [etc.], 1875; Jayawardhana, Ray. Strange New Worlds: The Search for Alien Planets and Life Beyond Our Solar System. Princeton, N.J.: Princeton University Press, 2011; Linde, Peter. The Hunt for Alien Life: A Wider Perspective. 1st edition 2016. Cham: Springer International Publishing: Imprint: Springer, 2016; Macvey, John W. Whispers From Space. New York: Macmillan, 1973; Morrison, Philip., John Billingham, and John Wolfe. TheSearch for Extraterrestrial Intelligence, SETI. [Washington, D.C.]: National Aeronautics and Space Administration, Scientific and Technical Information Office, 1977; Regis, Edward. Extraterrestrials: Science and Alien Intelligence. Cambridge [Cambridgeshire] ; New York: Cambridge University Press, 1985; Ross, Monte. The Search for Extraterrestrials: Intercepting Alien Signals. Berlin; New York : Chichester, UK: Springer; published in association with Praxis, 2009; SETI: Search for Extra-terrestrial Intelligence. Washington, D.C.: Moffett Field, Calif.: Pasadena, Calif: Washington, D.C.: National Aeronautics and Space Administration; Ames Research Center, SETI Office; Jet Propulsion Laboratory, California Institute of Technology, SETI Office; NASA Headquarters, Office of Space Sciences and Applications, Life Sciences Division, 1990; Shuch, H. Paul. Searching for Extraterrestrial Intelligence: SETI Past, Present, and Future. Berlin; Heidelberg; New York: Chichester: Springer; Praxis Publishing, 2011; Squeri, Lawrence. Waiting for Contact: The Search for Extraterrestrial Intelligence. Gainesville, Florida: University
Press of Florida, 2016; Traphagan, John. Extraterrestrial Intelligence and Human Imagination: SETI At the Intersection of Science, Religion, and Culture. Cham: Springer, 2014; Vakoch, Douglas A., and Albert A. Harrison. Civilizations Beyond Earth: Extraterrestrial Life and Society. New York: Berghahn Books, 2011; Waldrop, M. Mitchell. "The Search for Alien Intelligence: SETI Is Dead? Long Live SETI." Nature 475, no. 7357 (2011): 442-444; Wall, Michael. Out There: A Scientific Guide to Alien Life, Antimatter, and Human Space Travel (For the Cosmically Curious). Grand Central Publishing, New York, 2018.

27 Milan M. Cirkovic: The Great Silence: The Science and Philosophy of Fermi's Paradox: Oxford University Press, Great Clarendon Street, Oxford $\mathrm{OX}_{2}$ 6DP, United Kingdom, 2018; Davies, P. C. W. The Eerie Silence: Renewing Our Search for Alien Intelligence. 1st U.S. ed. Boston: Houghton Mifflin Harcourt, 2010; Webb, Stephen. If the Universe Is Teeming With Aliens ... Where Is Everybody?: Seventy-five Solutions to the Fermi Paradox and the Problem of Extraterrestrial Life. 2nd Ed. Springer, 2015.

28 Hippke, Michael. "Interstellar Communication: The Colors of Optical SETI." Journal of Astrophysics and Astronomy 39, no. 6 (2018): 1-16; Vakoch, Douglas A. Communication With Extraterrestrial Intelligence. Albany: State University of New York Press, 2011.

29 Haswell, Carole A. Transiting Exoplanets. Cambridge: Cambridge University Press, 2010.

30 Percival Lowell, Mars, Boston, Houghton, Mifflin, 1896, https://archive.org/details/ marsbypercivallooolowe/page/ n8; Mars and its canals, New York, The Macmillan company; 
London, Macmillan \& co., ltd., 1906, https://archive.org/details/ marsanditscanaloılowegoog/page/ n13; Mars as the Abode of Life, New York, The Macmillan company, 1908, https://archive.org/details/ agg9438.00o1.oo1.umich.edu/page/ n2, ultimo accesso 20 dicembre 2019.

31 H. G. Wells, The War of the Worlds, ultimo aggiornamento: 20 settembre, 2019, Project Gutenberg, https://www.gutenberg. org/files/36/36-h/36-h.htm; la trasmissione originale del 1938 è disponibile su vari siti tra cui: https://www.youtube.com/ watch?v=9q7tN7MhQ4 $\mathrm{I}$, ultimo accesso 16 dicembre 2019.

32 Ad esempio, Donald Keyhoe, Flying Saucers from Outer Space, New York, Henry Holt 1953.

$33 \mathrm{Si}$ veda ade esempio: Extraterrestrial life in popular culture https://en.wikipedia.org/ wiki/Category:Extraterrestrial_life_ in_popular_culture; lista di film con extraterrestri: https://en.wikipedia. org/wiki/List_of_films_featuring_ extraterrestrials; canzoni sulla vita extraterrestre: https://en.wikipedia. org/wiki/Category:Songs_about_ extraterrestrial_life; libri sulla vita extraterrestre: https://en.wikipedia. org/wiki/Category:Books_about_ extraterrestrial_life, ultimo accesso 20 dicembre 2019.

34 Achebe, Chinua. The African Trilogy. (London: Everyman's Library, 2010) ISBN 9781841593272. Edito con introduzione di Chimamanda Ngozi Adichie. Things Fall Apart, No Longer at Ease, e Arrow of God in un solo volume. 